\title{
XXXIV. Experiments and observations on the different modes in which death is produced by certain vegetable poisons
}

\section{B.C. Brodie Esq. F.R.S.}

To cite this article: B.C. Brodie Esq. F.R.S. (1811) XXXIV. Experiments and observations on the different modes in which death is produced by certain vegetable poisons, Philosophical Magazine Series 1, 38:161, 171-182, DOI: $10.1080 / 14786441108638625$

To link to this article: http://dx.doi.org/10.1080/14786441108638625

曲 Published online: 27 Jul 2009.

Submit your article to this journal $₫$

Џll Article views: 2

Q View related articles $₫$ 


\section{[ 171$]$}

XXXIV. Experiments and Olservations on the different Modes in which Death is produced by certain vegetalle Poisons. By B. C. Brodie, Esq. F.R.S. Communicated by the Society for promoting the Knowledge of Animal Chemistry.

[Concluded from p. 94.]

\section{Experiments with the Empyreumatic.Oil of Tobacco".}

Exp.13. Less than a drop of this oil was applied to the tongue of a young cat. Instantly violent convulsions took place in all the muscles, and the respirations became very frequent, In five minutes after the application, she lay on one side insensible, with slight spasmodic actions of the muscles. At the end of eleven minutes she retched, but did not vomit. In a quarter of an hour she appeared to be recovering. I repeated the application of the poison, and she was again seized with violent convulsions, and became insensible, breathing at long intervals ; and in two minutes from the second application respiration had entirely ceased, and she was apparently dead. On opening the thorax, I found the heart acting with regularity and strengtb, circulating dark-coloured blood. I introduced a tube into the trachea, and produced artificial respiration; the contractions of the heart became augmented in force and frequency, and there was no evident diminution in six or seven minutes, during which the artificial respiration was continued.

On dissection, nothing remarkable was found in the appearance of the tongue or brain.

The symptoms and mode of death, in this experiment, did not essentially differ from those produced by the essential oil of almonds. I was surprised to find the effects of the empyreumatic oil so entirely different from those of the infusion of tobacco. Supposing that this difference might arise from the poison being more concentrated in the oil than in the infusion, I made the following experiments.

Exp. 14. A drop of the oil of tobacco was suspended in an ounce and a half of water by means of mucilage of gum arabic, and the whole was injected into the rectum of a dog. In two minutes afterwards he became faint, retched, but did not vomit. He appeared to be recovering from this state, and in twenty-five minutes after the first injection it was

* I was furnished with the empyreumatic eil of tobacco by Mr. W. Brande. It may be procured by subjecting the leaves of tobacco to distillation in a heat above that of boiling water: a quantity of watery fluid comes over, on the surface of which is a thin film of unctuous substance. 
repeated in the same quantity. He was then seized with symptoms similar to those in the lastrexperiment, and in two minutes and a halt he was arparently dead.

Two minutes after apparent death, on the thorax being opened into, the heart was found acting regularly one hundred tines in a minute, and it continued acting for several minutes.

Exp. 15. A drop of the empyreumatic oil of tohacco with an ounce of water was injected into the rectum of a cat. The symptoms produced were in essential circumstances similar to those which occurred in the last experiment. The aninal was apparently dead in five minutes after the injection, and the heart continued to contract for several minutes afterwards.

We may conclude from these experiments, that the empyreumatic oil of tobacco, whether applied to the tongue or injected into the intestine, does not stop the action of the licart and induce srnoope, like the intusion of tobacco; but that it occasions death by destroying the functions of the brain, wihout directly acting on the circulation. In other words, its effects are similar to those of alcohol, the juice of aconite, and the essential oil of almonds.

\section{Experiments with Poisons applied io wounded Surfaces.}

Experiments with the Essential Oil of Almonds.

Exp. 16. I made an incision in the thigh of a rabbit, and introduced two drops of essential oil between the skin and the muscles. In four minutes after the application, he was seized with violent convulsions, and became insensible, and in two minutes more he was apparently dead; but the heart was felt through the ribs acting one hundred and twenty times in a minute, and it continued acting for several minutes. There were no other appearances in the limb, than would have resulted from an ordmary wound.

Exp. 17. Two drops of the essential vil of ahionds were introduced into a wound in the side of a mouse. Two minutes afterwards he was affected with symptoms similar to those which occurred in the last experiment, and in two minutes more he was apparently dead, but the heart continued to contract for some minutes afterwards.

From the experiments which I have just related, and from others which it appears unnecessary to detail, as the general results were the same, I have learned that where the essential oil of almonds is applied to a wound, its effects are not so instantaneous as when it is applied to the tongue; otherwise 
otherwise there is no difference in its effects, in whatever manner it is applied.

Experiments with the Juice of the Leaves of Aconite.

Exp. 18. I made a wound in the side of a young rabbit, and untroduced, between the skin and muscles, about twenty drops of the juice of aconite. Twenty-tbree minutes afterwards he was affected with sumptoms in all essential respects similar to those which occurred in an experiment already related, where the juice was injected into the rectum, and at the end of forty-seven minutes from the application of the poison he was apparently dead. Two minutes after apparent death, the heart was found contracting, but very feebly.

\section{Experiments with the Woorara*.}

Fxp. 19. A small quantity of the woorara in powder was applied to a wound in the side of a Guinea pig. Ten minutes afterwards the animal was unable to walk; then he became quite motionless, except some slight occasinnal convulsions. He gradually became insensible; the respirations were laboured, and at the end of fourteen minutes from the application of the poison the respiration had entirely ceased, and he was apparently dead; but on opening the thorax, the heart was found acting seventy limes in a minute, circulating dark coloured blood, and it comtinucd to contract for several minutes afterwards. On dissection no preternatural appearances were observed in the brain, nor was there any other appearance in the limb than would have arisen from an ordinary wound.

Exp. 20. I made a wound in the side of a Guinea pig, and introduced into it about wo grains of the woorara in powder. At the end of lwenty-five minutes, symptoms took piare very similar to those which occurred in the last experiment, and in thirteen minutes more the animal was apparently dead; but the heart continued to contract one hundred and eight times in a umute, and by means of artificial respiration the circulation was kept up for more than twenty ninutes.

The resulis of other experiments which I have made with the wnorara were similar to those just described. The beart continued to act after apparent death, and the circulation

* The VVoorara is a poison with which the Indiaus of Guiara arm the points of thcir arrows. It appears ikot w ditter escentialip from ine Ticulas, which was employed in the experiments of the sabe Fontana. I an indebted to Dr.E. $\mathrm{N}$ Bancroft, who not only fursished me with sone of the woorara which he had in his possession, but also lent me his assistance in the experiments which were made with it. 
might be kept up by means of artificial respiration. It is evident that this poison acts in some way or other on the brain, and that the cessation of the functions of this organ is the immediate cause of death.

I found in these experiments, that the best mode of applying the woorara is when it is dissolved in water to the consistence of a thin paste. I first made the wound, and then smeared the poison over it with the end of the scalpel. I found that the animal was more speedily and certainly affected, if there was some hæmorrhage, unless the hæmorrhage was very copious, when it produced an opposite effect, by washing the poison away from the wound. When the poison was applied in large quantity, it sometimes began to act in six or seven minutes. Never more than half an hour elapsed from the time of the poison being inserted, to that of the animal being affected, except in one instance, where a ligature was applied on the limb, which will be mentioned afterwards. The woorara, which I employed, had been preserved for some years, which will account for its having been less active than it has been described to be by those who had witnessed its effects when in a recent state.

\section{Experiments with the Upas Antiar*.}

Exp. 21. About two grains of this poison were made into a thin paste with water, and inserted into a wound in the thigh of a dog. Twelve minutes afterwards he became langiud; at the end of fifteen minutes, the heart was found to beat very irregularly, and with frequent intermissions; after this, he had a slight rigor. At the end of twenty minutes, the heart beat very feebly and irregularly; he was languid; was sick and vomited; but the respirations were as frequent and as full as under natural circumstances, and he was perfectly sensible. At the end of twenty minutes he suddenly fell on one side, and was apparently dead. I immediately opened into the thorax, and found the heart distended with blood in a very remarkable degree, and to have entirely ceased contracting. There was one distinct and full inspiration after I had begun making the incision into the thorax. The cavities of the left side of the heart contained scarlet blood, and those of the right side contained dark-coloured blood, as in a living animal.

Exp.22. A small quantity of the upas antiar, prepared

- We are informed that the island of Java produces two powerful vegetable poisons, to one of which the natives give the name of $U$ pas tieute, and to the other that of Upas antiar. I was supplied with a quantity of the latter through the kindness of Mr. Maryden, who had some of it in his possession. 
as before, was inserted into a wound in the thigh of a young ca1. She appeared languid in two minutes after the poison was inserted. The symptoms which took place did not essentially differ from those which occurred in the last experiment, except that there were some convulsive motions of the limbs. At eight minutes after the poison was inserted, she lay on one side motionless and insensible, the heart could not be felt, but the respiration had not entirely ceased. On opening into the thorax, I found the heart to have ceased contracting. It was much distended with blood: and the blood in the cavities of the left side was of a scarlet. colnur. There were two full inspirations after the incision of the thorax was begun. On irritating the heart with the point of the scalpel, slight contractions took place in the fibres of the appendices of the auricles, but none in any other part.

Exp. 23. The experiment was repeated on a rabbit. The symptoms produced were similar to those in the last experiment; but the animal did not vomit, and the convulsive motions were in a less degree : he died eleven minutes after the poison was inserted. On opening the chest, the beart was found to have entirely ceased contracting; it was much distended with blood; and the blood in the cavities of the left side was of a scarlet colour. On irritating the heart with the point of the scalpel, the ventricles contracted, but not sufficiently to restore the circulation.

Exp. 24. About a grain of the npas antiar was inserted into a wound in the side of a rabbit. He was affected with symptoms similar to those before described, and died in ten minutes after the poison was applied. On opening the thorax immediately after death, the heart was found to have ceased contracting, and the blood in the cavities of the left side was of a scarlet colour.

It appears from these experiments, that the upas antiar, when inserted into a wound, produces death (as infusion of tobacco does when injected into the intestines) by rendering the heart insensible to the stimulus of the blood, and stopping the circulation. The heart beats feebly and irregularly before either the functions of the mind or the respiration appear to be affected. Respiration is performed even after the circulation has ceased; and the lcft side of the heart is found after death to contain scarlet blood, which never can be the case where the cause of death is the cessation of the functions of the brain or lungs. The convulsions which occur when the circulation has nearly ceased, probably arise from the diminution of the supply of blood to the brain, resembling 
resembling those which take place in a pcrson who is dying from bæmorrhage.

Therc remains an interesting subject of inquiry, "through what medium do poisons infuence, the brain when applied to wounds?" That poisons applicd in this mamner do not produce thcir effects precisely in the same way as poisons taken internally, is rendered probable by this circumstance; that-some puisons, which are very powerful when applied to wounds even in small quantities, are either altogether inefficient when taken intertially, or require to be given in very large quantities, in order to produce their effect, and vice versâ.

A poison applied to a woinded surface may be supposed to act on the brain in one of three ways,

1. By means of the nerves, like poisons taken internally.

2. By passing into the circulation through the absorbent vessels.

3. By passing directly into the circulation through the divided veins.

Exp.25. In order to ascertain whether the woorara acts through the medium of the nerves, I exposed the axilla of a rabbit, and divided the spinal nerves supplying the upper extremity, just before they unite to form the axillary plexus. The operation was performed with the greatest care. I not only divided every nervous filament, however small, which I could detect, but every portion of cellular membrane in the axilla, so that the artery and vein were leftentirely in. sulated. I then made two wounds in the fore-arm, and inserted into them some of the woorara formed into a paste. Fourteen minutes after the poison was applied, the hind legs became paralytic, and in ten minutes more he died, with symptoms precisely sinilar to those which took place in the former experiments, and the heart continued to act after apparent death. On dissection, the nerves of the uppes. extremity were particularly examined, but not the smallest filament could be found undivided.

I made the following experimen1, to ascertain whether the woorara passes into the circulation through the absorbent vessels.

Exp. 26. I tied a ligature round the thoracic duct of a dog, just betore it perforates the angle of the left subclavian and jugular veins. I then made two wounds in the left hind leg, and introduced some of the woorara in powder into them. In less than a quatter of an hour be became affected with the usual symptoms, and died in a few minutes afterwards. 
After death, I dissected the thoracic duct with great care. 1 found it to have been perfectly secured by the ligature. It was very much distended with chyle; and about two inches below its termination its coats had given way, and chyle was extravasated into the cellular membrane. The lymphatic vessels in the left axilla were distended in a very remarkable degree; and on dividing them, not less than a drachm of lymph issued from the divided ends.

Since neither the division of the nerves nor the obstruction of the thoracic duct interfere in the shightest degree with the effects of the woorara, there is presumptive evidence that it acts on the brain by entering the circulation through the divided veins. I endeavoured to ascertain, by experiment, whether this is really the case.

To apply ligatures to the large vessels of a limb only would evidently lead to no satisfactory conclusion, since the anastomosing vessels might still carry on the circulation. The only way which I could rlevise of performing the cxperiment, was to include all the vessels, small as well as large, in a ligature.

Exp. 27. In order to make the experiment more satisfactorily, I exposed the sciatic nerve of a rabbit in the upper and posterior part of the thigh, and passed under it a tape half an inch wide. I then made a wound in the leg, and having introduced into it some of the woorara mixed with water, I tied the tape moderately tight on the fore-part of the thigh. Thus I interrupted the communication between the wounds and the other parts of the body, by means of the vessels, while that by means of the nerve still remained. After the ligature was tightened, I applied the woorara a second time, in another part of the leg. The rabbit was not at all affected, and at the end of an hour I removed the ligature. Being engaged in some otber pursuit, I did not watch the animal so closely as I should otherwise have done; but twenty minutes after the ligature was removed, I found him lying on one side, motionless and insensible, evidently under the inftuence of the poison; but the symptoms were less violent than in most instances, and after lying in this state he recovered, and the limb became perfectly warm, and he regained the power of using it.

Exp. 28. I repeated the last experiment with this difference, that after having applied the poison, I made the ligature as tight as I conld draw it. I removed the ligature at the end of an hour and twenty minutes, but the animal was not at all affected cither before or after the removal of the Vol. 38. No. 161. Sept. 1811 . M ligature, 
ligature, and on the following day he had recovered the use of the limb.

Exp.29. I repeated the experiment a third time, drawing the ligature very tight. At the end of forty-five minutes the animal continued perfectly well, and the ligature was removed. I watched him for three quarters of an hour afterwards, but there were no symptoms of his being affected by the poison. On the following day the rabbit died; but this I attribute to the injury done to the limb and sciatic nerve by the ligature, as there was the appearance of inflammation in the parts in the neighbourhood of the ligature.

These three experiments were made with the greatest care. From the mode in which the poison was applied, from the quantity employed, and from my prior experience, I should have entertained not the smallest doubt of the poison taking effect in every instance in less than twenty minutes, if no ligature had been applied. In two of the three, the quantity of woorara was more than had been used in any former experiments.

I have not judged it necessary to make any more experiments, with the ligature on the limb, because the numerous experiments of the Abbe Fontana on the ticunas, coincide in their results with those which have just been detailed, and fully establiah the ifficacy of the ligature in preventing the artion of the poison. II is not to be wondered at, "that the ligature should sometimes fail in its effects, since these must evitently depend on the derree in which the circulation is shstructed, and on the length of time during which the obstruction is continued.

There can be little doubt that the woorara affects the brain, by passing into the circulation through the divided vessels. It is probable that it dots not produce its effects, until it enters the substance of the frain, along with the blood, in which it is discolved; nor will the experiments of the Abbe Fontann, in which be found the ticunas produce almost instant dath when injceted into the jugular vein of a rabbit, be found to militate against this conclusion, when we consider how short is the distance which, in so small an animal, the blood has to pass from the jugular vein to the carotid artery, and the great rapidity of the circulation; since in a rabbit under the influence of terror, during such an experiment, the heart cannot be supposed to act so seldom as three times in a second.

$I$ have made no experiments to ascertain through what medium 
medium other poisons when applied to wounds affect the vital organs, but from analogy we may suppose that they enter the circulation through the divided blood-vessels.

IV.

The facts already related led me to conclude that alcohol, the essential oil of almonds, the juice of aconite, the oil of tobacco, and the woorara, occasion death simply by destroying the functions of the brain. The following experiment appears fully to establish the truth of this conclusion.

Exp. 30. The temperature of the room being $58^{\circ}$ of Fahrenheit's thermometer, I made two wounds in the side of a rabbit, and applied to them some of the woorara in the form of paste. In seven minutes after the application, the hind legs were paralysed, and in fiften minutes respiration had ceased, and he was apparently dead. Two minutes afterwards the heart was still beating, and a tube was introduced through an opening into the tracliea, by means of which the lungs were inflated. The artificial respiration was made regularly about thirty-six times in a minute.

At first, the heart contracted one hundred times in a minute.

At the end of forty minutes, the pulse had risen to one handred and twenty in a minute.

At the end of an hour, it had risen to one hundred and forty in a minute.

At the end of an hour and twenty-three minutes, the pulse had fallen to a hundred, and the artificial respiration was discontiuued.

At the commencernent of the experiment, the ball of $\mathbf{a}$ thermometer being placed in the rectum, the quicksilver rose to one hundred degrees; ai the close of the experiment it had fallen to eighty-eight and a half.

During the cuntinuance of the artificial respiration, the blood in the femoral artery was of a florid red, and that in the femoral vein of a dark colour, as usual.

It has been observed by M. Bichat, that the immediale cause of death, when it takes place suddenly, must be the cessation of the functions of the heart, the brain, or the lungs. This observation may be extended to death under all circumstances. The stomach, the liver, the kidneys, and many other organs are necessary to life, but their constant action is not necessary; and the cessation of their functions cannot therefore be the immediate cause of death, As in this case the action of the heart had never ceased; as the cifculation of the blood was kept up by artificial respi- 
ration for more than an hour and twenty minutes after the poison had produced its full effects; and as during this time the usual cbanges in the colour of the blood took place in the lungs; it is evident that the functions of the heart and lungs were unimpaired: but that those of the brain had ceased, is proved by the animal having continued in a state of complete insensibility; and by this circumstance, that animal heat, to the generation of which I have formerly shown the influence of the brain to be necessary, was not generated.

Having learned that the circulation might be kept up by artificial respiration for a considerable time after the woorara had produced its full effects, it occurred to me that in an animal under the influence of this or of any other poison that acts in a similar manner, by continuing the artificial respiration for a sufficient length of time after natural respiration had ccased, the brain might recover from the impression which the prison had produced, and the animal might be restored to life. In the last experiment, the animal gave no sign of returning sensibility; but it is to be observed, 1. That the quanrity of the poison employed was very large. 2. That there was a great loss of animal heat, in consequence of the temperature of the rom being much below the natural temperature of the auimal, which could not thercfore be considered uuder such favourable circumstances as to recovery, as if it had been kept in a higher temperature. 3. That the circulation was still vigorous when l left off infling the lungs, and therefore it camnot be kuown what would have been the result, if the artificial respiration had been longer continued.

Exp. 30. A wound was male in the side of a rabbit, and one drop of the esscutial ail of almonds was inserted into it, and immediately the animal was placed in a temperature of $90^{\circ}$. In two minutes le was under the intluence of the poison. The usual symptoms took place, and in three miuntes more respiration had ceased, and he lay apparently icad, but the heart was still felt beating through the ribs. $A$ tube was then introduced into one of the nostrils, and the lungs were inflated about thirty-five times in a minute. Six minutes after the commencement of artificial respiration, be moved his head and legs, and made an effort to breathe. He then was seized with convulsions, and again lay motionless, but continued to make occasional efforts to breathe. Sixteen ninutes after its commencement, the artificial respriration was discontinued. He now breathed spontaneously seventy times in a minute, and moved his 
head and extremities. After this, he occasionally rose, and attempted to walk. In the intervals he continued in a dozing state; but from this he gradually recovered. In less than two hours he appeared perfectly well, and he continued well on the following day.

The inflating the lungs has been frequently recommended in cases of suffocation, where the cause of death is the ces. sation of the functions of the lungs: as far as $1 \mathrm{know}$, it has not been before proposed in those cases, in which the cause of death is the cessation of the functions of the brain*. It is probable that this method of treatment might be employed with advantage for the recovery of persons labouring under the effects of opium, and many other poisons.

\section{V.}

The experiments which have been detailed lead to the following conclusions.

1. Alcohol, the essential oil of almonds, the juice of aconite, the empyreumatic oil of tobacco, and the woorara, act as poisons by simply destroying the functions of the brain; universal death laking place, because respiration is under the influence of the brain, and ceases when its functions are destroyed.

2. The infusion of tobacco when injected into the intes tine, and the upas antiar when applied to a wound, have the power of rendering the heart insensible to the stimulus of the blood, thus stopping the circulation; in other words, they occasion syncope.

3. There is reason to believe that the poisons, which in these experiments were applied internally, produce their effects through the medium of the nerves without being absorbed into the circulation.

4. When the woorara is applied to a wound, it produces its effects on the brain, by entering the circulation through the divided blood-vessels, and, from analogy, we may conclude that other poisons, when appliced to wounds, operate in a similar manner.

5. When an animal is apparently dead from the influence of a poison, which acts by simply destroying the functions

* Since this paper was read, I have been favoured by the Right Fion. the President with the perusal of a Dissertation on the Efrects of the Upas 'Tieut $\dot{e}$, lately published at Paris by $M$ Delile, by which I find that he had employed artificial respiration for the purpose of recovering animals, which were under the infuence of this poison, with success. M. Delile describes the Upas Tieutè as causing death, by occasioning repeated and loig-continued con. tractions of the muscles of respiration, on which it acts through the medium of the spinal marrow, without destroying the functions of the brain.

M 3 
of the brain, it may, in some instances at least, be made to recover, if respiration is artificially produced, and continued for a certain length of time.

From analogy we might draw some conclusions respecting the mode in which some other vegetable poisons produce their effects on the animal system; but I forbear to enter into any speculative inquiries; as it is my wish, in the present communication, to record such facts only, as appear to be established by actual experiment.

\section{Addition to the Croonian Lecture for the Year 1810.}

In the experiments formerly detailed, where the circulation was maintained by means of artificial respiration after the head was removed, I observed that the blood, in its passage through the lungs, was altered from a dark to a scarlet colour, and hence I was led to conclude that the action of the air produced in it changes analogous to those which occur under ordinary circumstances. I have lately, with the assistance of my friend $\mathrm{Mr}$. W. Brande, made the following experiment, which appears to confirm the truth of this conclusion.

An elastic gum bottle, having a tube and stop-cock connected with it, was filled with about a pint of oxygen gas. The spinal marrow was divided in the neck of a young rabbit, and the blood-vessels having been secured, the head was removed, and the circulation was maintained by inflating the lungs with atmospheric air for five minutes, at the end of which time the tube of the gum bottle was inserted into the trachea, and carefully secured by a ligature, so that no air might escape. By making pressure on the gum bottle, the gas was made to pass and repass into and from the lungs about thirty times in a minute. At first, the heart acted one hundred and twenty times in a minute, with regularity and strength; the thermometer, in the rectum, rose to $100^{\circ}$. At the end of an hour, the heart acted as frequently as before, but more feebly; the blood in the arteries was very little more florid than that in the veins; the thermometer in the rectum had fallen to $93^{\circ}$. The gum bottle was then removed. On causing a stream of the gas which it contained to pass through lime-water, the presence of carbonic acid was indicated by the liquid being instantly rendered turbid. The proportion of carbonic acid was not accurately determined; but it appeared to form about one-half of the quantity of gas in the bottle.

B. C. Brodie.

$\mathrm{XXXV} \cdot A n$ 